\title{
Helmuth Rolfes Gibt es eine kirchenamtliche Lehre der sozialen Kommunikation? \\ Ein Rückblick 40 Jahre nach "Inter Mirifica"
}

Auf fast allen Ebenen kirchlichen Lebens - und zwar weltweit - gibt es inzwischen vielfältige Institutionalisierungen kirchlicher Medienarbeit, angefangen beim Päpstlichen Rat für die Sozialen Kommunikationsmittel, über Einrichtungen der Bischofskonferenzen und einzelner Bistümer bis hin zu Ausschüssen für Medien- und Öffentlichkeitsarbeit auf der Ebene der Pfarreien. Alle diese Einrichtungen sollen das kirchliche Medienengagement fördern und anleiten, es koordinieren und strukturieren, aber auch dem kirchlichen Sendungsauftrag entsprechend regulieren.

Gibt es für diese in unseren modernen Medien- und Informationsgesellschaften hochkomplexe Aufgabe kirchenamtliche Orientierungshilfen, Anweisungen oder auch Lehraussagen, denen in Zukunft vielleicht eine ähnliche Bedeutung für die Entwicklung einer kirchlichen Lehre der sozialen Kommunikation zukommen könnte, wie den päpstlichen Sozialenzykliken des 19. und 20. Jahrhunderts für die christliche Gesellschaftslehre?

Die Frage lässt sich nicht abstrakt beantworten, sondern nur im Durchgang durch die kirchlichen Verlautbarungen selbst. Ein solcher Durchgang soll im Folgenden versucht werden, wobei als Ausgangspunkt der Analyse das Konzilsdekret „Inter Mirifica“ (IM) des Zweiten Vatikanischen Konzil gewählt wird. Danach werden die nachfolgenden kirchenamtlichen Dokumente, insofern sie den Bereich der sozialen Kommunikation ausdrücklich thematisch behandeln, untersucht. Vorher soll aber kurz begründet werden, warum gerade das Zweite Vatikanische Konzil als Ausgangspunkt für die Beantwortung der Frage, ob es eine eigene Lehre der Kirche zur sozialen Kommunikation gibt, gewählt wird.

\section{Das Zweite Vatikanische Konzil als Ausgangspunkt}

Natürlich könnte man zur Beantwortung der Themenfrage auch sehr viel weiter in die Geschichte der Kirche zurückgehen. Schließlich sind der christliche Glaube und die Kirche von ihrem Ursprung her mit dem Medienthema verbunden. Das Christentum ist eine Buchreligion, denn die entscheidende Norm des Glaubens und des kirchlichen Handelns ist in der Heiligen Schrift grundgelegt, also in einer Sammlung von Texten. Dass die Kirche in ihrem Einflussbereich deshalb seit jeher mit besonderer Aufmerksamkeit die Erstellung, Verteilung und Lektüre von Texten 
verfolgte, zumal wenn in ihnen Fragen der Religion oder Ethik behandelt wurden, muss niemanden verwundern und tatsächlich gibt es auch eine lange und wechselvolle Geschichte der Kirche im Umgang mit den Büchern.

In der frühen Zeit der Kirche befassten sich bereits Konzilien mit der Ordnung und Anerkennung der für kanonisch eingestuften Schriften und verboten so genannte häretische Schriften. In Apg 19,19 kann man sogar schon von einer Bücherverbrennung lesen: „Und nicht wenige, die Zauberei getrieben hatten, brachten ihre Zauberbücher herbei und verbrannten sie vor aller Augen." Diese Schriftstelle musste immer wieder dazu herhalten, Zensur- und Unterdrückungsmaßnahmen der kirchlichen Obrigkeit zu rechtfertigen. So findet sich in verschiedenen Ausgaben des römischen Index ein ausdrücklicher Hinweis auf diese Schriftstelle ${ }^{1}$, und noch 1904 schrieb Joseph Hilgers in seiner großen Arbeit über den Index über den Eifer des Völkerapostels Paulus, „mit dem er nach dem Zeugnis der Apostelgeschichte zu Ephesus die abergläubischen Bücher unter seinen Augen verbrennen ließ". ${ }^{2}$ Die Bischöfe der alten Kirche hätten „eingedenk dieses apostolischen Beispiels alle Anstrengungen unternommen, um ihrerseits mit allen Mitteln heidnische und häretische Bücher von den Gläubigen fernzuhalten“.3

Ganz offensichtlich wird von Hilgers an dieser Stelle eine Praxis in die apostolische Zeit zurückprojiziert, die erst möglich wurde, als sich das Christentum als Staatsreligion durchsetzte. Seit dieser Zeit wurden Besitz und Lektüre häretischer Schriften tatsächlich auf dem Wege über kaiserliche Edikte unter Strafe gestellt. In der frühen Kirche wurden kirchenamtliche Verfügungen zur Abwehr von Irrlehren dagegen „nur

1 Heinrich Reusch, Der Index der verbotenen Bücher, Bonn 1883 - 1885, Bd. 1, 8 .

2 Joseph Hilgers, Der Index der verbotenen Bücher, Freiburg 1904, 3.

3 Ebd. 3. Nun wird mit einer solchen Interpretation freilich der Sinn von Apg 19,19 auf den Kopf gestellt. Vgl. Rudolf Pesch, Die Apostelgeschichte, EKK Bd. V/2, 73 f. Die Neubekehrten in Ephesus folgen nicht einer Anordnung des Paulus, sondern bringen als Konsequenz ihrer Bekehrung freiwillig ihre Zauberbücher. Sie verbrennen diese Zauberbücher „vor allen", d.h. also vor der Gemeinde, um auf diese Weise zum Ausdruck zu bringen, das christliches Leben ohne Zauber und Magie auskommt (Vgl. EKK Peschner 73f.). Das hat mit einer amtlich angeordneten und durchgeführten Bücherverbrennung nichts zu tun. Es ist übrigens auch Hilgers aufgefallen, dass der Text nicht sagt, Paulus habe die Verbrennung angeordnet. Um aber seine Auffassung vom Recht und der Pflicht der kirchlichen Obrigkeit zur Bücherzensur biblisch weiterhin $z u$ legitimieren, interpretiert er Apg in seinem Sinne: „Denn auch wenn es nicht auf sein [Paulus] Geheiß geschah, billigte er zweifelsohne die Verbrennung und hieß sie gut, ja sah darin eine besondere Wirkung der Gnade Gottes.“ Ebd. 
durch Belehrung und Ermahnung"4 zur Geltung gebracht. Als das Christentum unter Konstantin zur Staatsreligion avancierte, erhielt der kirchliche Umgang mit häretischen Schrifttum eine neue Qualität. In dem $\mathrm{Maße}$, in dem die Macht der Kirche wuchs, verfestigte sich auch ihre Monopolstellung in der mit staatlich ausgeführten Strafandrohungen verknüpften Kontrolle von Büchern. Bis zur Erfindung des Buchdrucks konnte die Kirche diese Monopolstellung und damit verbunden auch ihre Kontrollmöglichkeiten in der Produktion von Schriften, die in den Schreibstuben der Klöster erfolgte, sowie in der Verteilung und Lektüre weitgehend unangefochten aufrechterhalten und durchsetzen.

Mit der Erfindung des Buchdrucks entstand eine völlig neue Lage. Das kirchliche Monopol war mit dieser Erfindung nämlich auf der Ebene der Produktion von Büchern gebrochen und auch die Distribution und Konsumption konnten aufgrund des Zerfalls der konfessionellen Einheit der Gesellschaft durch die Reformation nicht mehr in der bisherigen Weise letztlich kontrolliert und geregelt werden. ${ }^{5}$

Die kirchliche Obrigkeit reagierte auf die veränderte Lage umgehend mit entsprechenden Maßnahmen. ${ }^{6}$ Kirchenrechtliche Regelungen, Zensurmaßnahmen, die Indizierung missliebiger Bücher und auch inquisitorische Verfolgung prägten in der Folgezeit weitgehend die Methoden, mit denen die Kirche versuchte, unter den neuen Bedingungen in ihrem Sinn Produktion, Distribution und Konsumption von Büchern und Schriften zu regulieren. Wenigstens im Herrschaftsbereich der eigenen Konfession sollte das alte Monopol weiterhin aufrechterhalten werden. An der Geschichte des Index bis zu seiner späten Aufhebung im Umfeld des Zweiten Vatikanischen Konzils lässt sich diese Praxis der Kirche nachbuchstabieren. ${ }^{7}$ Es war eine Praxis, die im Zuge der neuzeitlichen Freiheitsbewegungen freilich immer anachronistischer und auch erfolgloser wurde und mit der die Kirche sich im Medien- und Kommunikationsbereich in eine durch Misstrauen und Abwehr gekennzeichnete, nach rückwärts gewandte und unfruchtbare Ungleichzeitigkeit mit der modernen Welt hineinmanövrierte.

Es war das Zweite Vatikanische Konzil, das in einer grundlegenden

Reusch, a.a.0., 9.

5 Vgl. Bernd Moeller, Flugschriften der Reformationszeit, in: TRE Band XI, Berlin, New York, 1983, 240-246.

6 Ein Beispiel ist die Konstitution Inter Multiplices von Innozens VIII. vom 17 November 1487. Diese Konstitution ist abgedruckt in: Arthur Utz/Brigitta von Galen (Hrsg.), Die katholische Sozialdoktrin in ihrer geschichtlichen Entfaltung. Bd. 2. Aachen 1976, 1524-1529.

7 Vgl. Georg May, Die Aufhebung der kirchlichen Bücherverbote,in: Ecclesia et ius, FS A. Scheuermann, München 1968, 547-571. 
Neubestimmung des Verhältnisses von Kirche und Welt mit diesem Erbe brach und damit eine neue Ära im Verständnis von sozialer Kommunikation und im Umgang mit den Medien einleitete. Dass schon vor dem Zweiten Vaticanum und hier besonders unter dem Pontifikat Pius XII. entscheidenden Weichenstellungen in der kirchlichen Medienarbeit und in der Einschätzung von Öffentlichkeit für eine freiheitliche und demokratische Gesellschaft vorgenommen wurden, die auch in die Überlegungen des Konzils Eingang gefunden haben, soll ausdrücklich erwähnt werden. ${ }^{8}$

\section{Ein konziliares Dekret über die sozialen Kommunikationsmittel}

\subsection{Der Arbeitsauftrag}

Am 4. Dezember 1963 wurde das Dekret über die sozialen Kommunikationsmittel "Inter Mirifica“ vom II. Vatikanischen Konzil beschlossen und unmittelbar darauf vom Papst in Kraft gesetzt. ${ }^{9}$ Im Rückblick ist es schon einigermaßen verwunderlich, dass das Konzil. erstmals in der $\mathrm{Ge}$ schichte der Kirche ein eigenes Dekret über die sozialen Kommunikationsmittel, denn so werden die Medien in der Sprache des Konzils genannt, verabschiedete.

Die überwältigende Anzahl der Bischöfe, der Ordensoberen und der Kirchlichen Hochschulen hatten im Vorfeld des Konzils das Thema der sozialen Kommunikationsmittel sicher nicht auf ihrer Wunschliste. Das zeigt schon das Ergebnis der Umfrage der vom Papst für das angekündigte Konzil eingerichteten Vorbereitungskommission (commissio antepraeparatoria): Auf der Grundlage der insgesamt 2150 Antwortschreiben formulierte die Kommission 9348 verschiedene Themenanträge für eine eventuelle Behandlung auf dem Konzil. Lediglich 18 dieser Anträge betrafen die Kommunikationsmittel. Während hinter 15 Anträgen etwa gut

8 Papst Pius XII. hat sich während seines langen Pontifikates in Reden, Radiobotschaften, Grußadressen etc. immer wieder zu Fragen der sozialen Kommunikation und Medien geäußert. Von besonderer Bedeutung ist die Enzyklika Miranda Prorsus über Film, Funk und Fernsehen vom 8.9.1957.

9 Eine umfassende Aufarbeitung der Entstehungsgeschichte und Bedeutung von Inter Mirifica findet sich bei Enrico Baragli, L'Inter Mirifica - Introduzione, Storia, Discussione, Commento, Documentazione. Rom 1969. Weiterhin vgl. Karlheinz Schmidthüs, Einleitung und Kommentar zum Dekret über die sozialen Kommunikationsmittel, in: Das Zweite Vatikanische Konzil I, LThK, Freiburg 1966, 112-135. Otto B. Roegele, Das Konzilsdekret „Über die Werkzeuge der sozialen Kommunikation“, in: Publizistik 9 (1964), 305-347. 
80 Bischöfe (davon fünf deutsche Bischöfe) standen, kamen die drei anderen Anträge aus dem Kreis der Ordensoberen. ${ }^{10}$

Trotz dieser im Grunde schmalen Antragslage sah dann das motu proprio „Superno Dei nutu“ vom 5. Juni 1960, durch das Kommissionen zur Erarbeitung von Textvorlagen für das kommende Konzil errichtet wurden, auch ein Sekretariat vor, das den Auftrag erhielt, im Laufe der nun folgenden zweiten Vorbereitungsphase des Konzils (Juni 1960 bis Herbst 1962) ein Schema über die Kommunikationsmittel zu erarbeiten.

Der zu erarbeitende Text solle - so lautete der Arbeitsauftrag ${ }^{11}$ - eine Zusammenfassung der kirchlichen Lehre über die sozialen Kommunikationsmittel bieten; er solle die Gewissensbildung im richtigen Gebrauch der Medien fördern, den Zusammenhang des Medienbereichs mit den Anforderungen von Glaube und Sitte herausstellen, und er solle den Einsatz der Medien für die Verkündigung behandeln. In diesem Arbeitsauftrag spricht sich die Überzeugung aus, dass es so etwas wie eine eigene kirchliche Lehre zur sozialen Kommunikation gebe und diese in den Grundzügen auch in dem geplanten Konzilstext vorgestellt werden soll.

Betrachtet man nun die Genese und den endgültigen Text des Dekretes, so finden wir eine erste, allerdings noch sehr vorläufige Antwort auf die Frage nach einer kirchenamtlichen Lehre zur sozialen Kommunikation.

\subsection{Das Ergebnis}

Im August 1962 erhielten die Konzilsväter dann einen 114 Abschnitte umfassenden Textentwurf der in der ersten Sitzungsperiode am 23.11.1962 vom Konzil beraten und nach kurzer Debatte ${ }^{12}$ mit folgenden Auflagen zur weiteren Bearbeitung an die Kommission zurückverwiesen wurde: Der neue Text solle in einer stark gekürzten Form nur noch die wesentlichen lehrhaften Grundsätze und die allgemeineren pastoralen Leitlinien aufführen. Praktische Fragestellungen sollten dagegen nicht mehr im Dekret selbst, sondern von einer vom Dekret eigens vorzusehenden Pastoralinstruktion durch eine nachkonziliare Kommission bearbeitet werden.

10 Enrico Baragli, a.a.0., $94 \mathrm{f}$.

11 Vgl. Karlheinz Schmidthüs, a.a.0., 112.

12 Vgl. dazu Xavier Rynne, Die zweite Reformation. Die erste Sitzungsperiode des Zweiten Vatikanischen Konzils. Entstehung und Verlauf. Köln, Berlin 1964, 201215 (Die Debatte über die „Kommunikationsmittel“). Otto B. Roegele (a.a.0., 310) hat nachgerechnet, dass die Väter sich für die Debatte insgesamt lediglich 360 Minuten Zeit genommen hatten. Offensichtlich betrachteten viele das Thema im Vergleich $z \mathfrak{u}$ den anderen Themen des Konzils als theologisch nicht so wichtig. 
Auf der Grundlage dieser Vorgaben entstand daraufhin ein nunmehr auf neun Druckseiten gekürzter Text, der nach kurzer Beratung und ohne gravierende Änderungen am 25.11.1963 vom Konzil endgültig beschlossen wurde. ${ }^{13}$ Der verabschiedete Text enthält neben einem Vorwort und einer Schlussbemerkung nur noch zwei Kapitel. Das Vorwort beginnt mit der Kennzeichnung der Medien als „soziale Kommunikationsmittel" (1) und weist auf die Chancen ihres rechten Gebrauchs, aber auch auf die Gefährdungen durch Missbrauch hin (2).

Im ersten Kapitel geht es um lehrhafte Aussagen und um sittliche Weisungen. Eröffnet wird das Kapitel mit der Feststellung des ursprünglichen Rechtes der Kirche, die sozialen Kommunikationsmittel zu benutzen und zu besitzen (3), und mit der Feststellung, dass ihr rechter Gebrauch vor allem die Kenntnis und Respektierung der Grundsätze der sittlichen Wertordnung sowie die Einsicht in die Eigengesetzlichkeit der Medien erfordert (4). Für den Gebrauch der sozialen Kommunikationsmittel sind „klare Gewissensgrundsätze“ (5) notwendig. Diese werden als erstes für den Bereich "Information" angesprochen. Es gibt in der menschlichen Gesellschaft ein „Recht auf Information“, dass sich aber an den Erfordernissen der Wahrheit und an der Achtung vor der Würde des Menschen zu orientieren hat (5). Sodann wird zweitens der Vorrang der sittlichen Ordnung vor der Freiheit der Kunst herausgestellt (6) und drittens eine jede Darstellung des Bösen durch die Medien den ethischen Forderungen untergeordnet (7). Es folgt eine Bemerkung zur Bedeutung „öffentlicher Meinungen" und zu der Verpflichtung, zur Bildung und Verbreitung richtiger öffentlicher Meinungen beizutragen (8). Die nächsten Abschnitte befassen sich mit der Verantwortung der Rezipienten im richtigen Gebrauch der Medien (9), mit der Erziehung, besonders der Jugendlichen, zu „Zucht und Maß" beim Gebrauch der sozialen Kommunikationsmittel (10) und mit der besonderen Verantwortung all derer, die an ihrer Produktion und Verbreitung beteiligt sind. Erwähnt wird die Verantwortung für das Gemeinwohl, für die Einhaltung der moralischen Richtlinien und für den besonderen Schutz der Jugendlichen (11). Abgeschlossen wird das erste Kapitel mit dem Verweis auf die besonderen Verpflichtungen der öffentlichen Gewalt, die Freiheit, und hier besonders die Pressefreiheit, zu schützen, wertvolle Aktivitäten im Medienbereich zu fördern sowie den Missbrauch der sozialen Kommunikationsmittel zu unterbinden und besondere Schutzmaßnahmen für Jugendliche $z u$ treffen (12).

Das zweite Kapitel beginnt mit dem Hinweis auf die Verpflichtung, die

13 Vgl. Karlheinz Schmidthïs, a.a.0., 112 f. 
sozialen Kommunikationsmittel für das Apostolat und die Verkündigung einzusetzen (13). Es folgt der Aufruf zur Förderung der guten Presse und eine Aufgabenzuweisung der katholischen Presse. Sie habe die Aufgabe, die „offentlichen Meinungen $z \mathfrak{u}$ bilden, $z \mathfrak{u}$ festigen und $z \mathfrak{u}$ fördern, die mit dem Naturrecht und den katholischen Lehren und Grundsätzen übereinstimmen" und Informationen über das kirchliche Leben $z \mathfrak{u}$ übermitteln und zu kommentieren. (14). Sodann spricht der Text über die Notwendigkeit der Schaffung und Vermehrung von qualifizierten Ausbildungsstätten für alle im Bereich der sozialen Kommunikationsmittel Tätigen (15) sowie von der Bedeutung der verschiedenen von christlichem Geist geprägten Bildungsangebote für den rechten Umgang und das Verständnis sozialer Kommunikationsmittel (16). Er fordert die ausreichende finanzielle Ausstattung entsprechender Medieneinrichtungen und erinnert an die Verpflichtung der Gläubigen, die katholische Medienarbeit auch finanziell zu unterstützen (17). Die letzten Abschnitte des zweiten Kapitels befassen sich mit institutionellen Fragen: Es soll in der gesamten Kirche ein besonderer Tag der sozialen Kommunikationsmittel festgesetzt werden (18), für den Papst soll beim Apostolischen Stuhl eine eigene Stelle für die sozialen Kommunikationsmittel zur Verfügung stehen (19); den einzelnen Bischöfen wird aufgetragen, in ihren Diözesen die Werke und Unternehmungen im Bereich der sozialen Kommunikation $z \mathfrak{u}$ fördern und $z \mathfrak{u}$ koordinieren (20); für die Landesebene ordnet das Konzil eigene nationale Medienstellen unter Leitung eines Bischofs oder der Bischofskonferenz an (21), die ihrerseits in enger Abstimmung mit den unter ausschließlicher Leitung des Apostolischen Stuhles stehenden jeweiligen internationalen Verbänden zusammenarbeiten sollen (22).

Im Schlusswort wird „im ausdrücklichen Auftrag des Konzils“ ein Pastoralschreiben angekündigt, das von der dem Apostolischen Stuhl unterstehenden Einrichtung unter Hinzuziehung von Sachverständigen aus verschiedenen Ländern erarbeitet werden soll (23). Der Text schließt mit einem Appell, die in dem Dekret genannten Grundsätze und Weisungen anzunehmen und umzusetzen (24).

An dieser Stelle müssen zwei öffentlichkeitswirksame Initiativen ${ }^{14}$ erwähnt werden, die auf erhebliche Mängel des Textes aufmerksam machten und die darauf abzielten, noch im letzten Moment eine erneute Beratung und Verbesserung des Textes zu erwirken. Am 16.11.1963 veröffentlichte eine Gruppe amerikanischer Journalisten eine Stellungnahme, der sich auch einige bekannte Konzilstheologen anschlossen. Am 
Tag darauf formulierten 90 Konzilsväter, unter ihnen theologisch hoch angesehene Bischöfe und Kardinäle, einen Antrag an die Konzilskommission, das Schema erneut zu überprüfen. Es genüge in dèr jetzigen Fassung nicht den Anforderungen, die an einen Konzilstext zu stellen seien. Diese Petition der Bischöfe wurde mit Verweis auf die Geschäftsordnung vom zuständigen Vorsitzenden der Kommission, Kardinal Cento, abgewiesen.

Was waren nun die hauptsächlichen Kritikpunkte? Die journalistischen Fachleute monierten den lebensfremden, abstrakten Stil des Textes, der mit der tatsächlichen journalistischen Arbeit nichts zu tun hätte. Sie bemängelten die moralistische Überformung legitimer kultureller und künstlerischer Ausdrucksformen, die dadurch in ihrem eigenständigen Wert verkannt würden. Sie kritisierten die halbierte Sichtweise des im Text angesprochenen Rechts auf Information, insofern nur die Übermittler, nicht aber die Verwalter bzw. die Quellen der Information in den Blick genommen würden. Die Vorstellung, die katholische Presse könne die Anforderungen des Naturrechts journalistisch quasi unfehlbar umsetzen, wurde ebenso kritisiert wie die kirchlichen Autoritätsansprüche gegenüber Publizisten und publizistischen Arbeitgebern. Schließlich fand auch die im Text beschriebene Aufgabenzuweisung der öffentlichen Gewalt gegenüber den Medien bei den journalistischen Kritikern keine Zustimmung, da dadurch die notwendige Freiheit für die Presse nicht mehr ausreichend gesichert sei. Das Dokument werde später einmal nach der Prognose der Kritiker - als Beispiel für eine verfehlte Wahrnehmung der Lebenswelt in einer die Menschen elementar berührenden Frage dastehen. Eigentlich, so die unausgesprochene Konsequenz dieser fundamentalen Kritik der Fachleute, müßte das Schema völlig neu überarbeitet werden, solle es den berechtigten Erwartungen der modernen Zeit genügen. ${ }^{15}$

Die im Antrag an die Konzilskommission vorgetragene Kritik der 90 Bischöfe hatte einen etwas anderen Tenor, war aber nicht minder radikal und endete in der Feststellung, es sei besser kein Dekret als dieses zu verabschieden. Für die Fachleute, aber auch für die berechtigten Erwartungen der Christen, sei das Dekret ungenügend und würde, sollte es in der jetzigen Form verabschiedet werden, das Ansehen des Konzils schädigen. Die fünf zentralen Punkte aus dem Antragstext seien hier wörtlich zitiert. Sie lauten: „1. Das Schema geht von unleugbaren Rechten der Kirche aus; es schweigt aber von der Grundlage jeder Kommunikation, nämlich dem Streben nach Wahrheit und dem Drang, sie auszusa-

15 Der Text dieser Kritik ist dokumentiert bei Enrico Baragli, a.a.O., Tav. XXIII. 
gen. 2. Deshalb wird das Wesen der Kommunikation nicht gebührend berücksichtigt. Die Kommunikationsmittel werden im technischen Sinne als Mittel der bloßen Anrede beschrieben, weniger als Mittel wahrer Kommunikation, d. h. eines eigentlich menschlichen Gesprächs. 3. Vergeblich sucht man im Schema die Darlegung des Gedankens der Bildung einer wahren christlichen Humanität, durch die der Mensch angeleitet wird, nicht bloß das Sensationelle wahrzunehmen, sondern sein Verlangen auszubilden, die Wahrheit zu hören und auf richtige Weise zu sehen. Eine solche Bildung vermeidet die Distanz des rein abstrakten Wissens und Urteilens und führt den Menschen zur wahren Kommunikation, durch die er teilhaftig gemacht wird am Schicksal anderer. 4. Am meisten ist $\mathrm{zu}$ bedauern, dass den Laien der ihnen zukommende Platz nicht zuerkannt wird, so dass sie auch dort, wo sie zuständiger sind als die Kleriker, das Gefühl haben müssen, unter klerikaler Vormundschaft gehalten zu werden. 5. Viele Fragen, die im Schema berührt werden, können nicht in allgemeingültiger Weise entschieden werden. Sie gehören weniger auf ein Ökumenisches Konzil als auf nationale oder regionale Bischofskonferenzen." 16

Diese durchaus berechtigte Kritik, die in der Konsequenz tatsächlich eine Neuerarbeitung des Textes notwendig gemacht hätte, hat keinen Eingang mehr in den Text des Dekretes gefunden. Sie hat aber offensichtlich deutlich das Abstimmungsverhalten der Konzilsväter beeinflusst. Kein anderer Text wurde nämlich mit so vielen ablehnenden Stimmen verabschiedet, wie das Dekret „Inter mirifica“. In der Abstimmung am 25.11.1963 über das ganze Schema stimmten von 2112 Anwesenden lediglich 1598 mit Ja , 503 mit Nein, 11 Stimmen waren ungültig. Ein so schwaches Endergebnis hat es bei keinem anderen Konzilsdokument gegeben. Und selbst bei der letzten, formalen und feierlichen Endabstimmung vom 4.12.1963 unter Leitung des Papstes stimmten 1960 Stimmberechtigte mit $\mathrm{Ja}$, aber immerhin 164 noch mit Nein und 27 enthielten sich. ${ }^{17}$

\subsection{Die Bedeutung von Inter Mirifica für die Entwicklung einer kirchlichen Lehre zur sozialen Kommunikation}

IM gehört nicht zu den herausragenden Texten, die vom Konzil erarbeitet und verabschiedet wurden. Entsprechend war auch das Echo unter Fach-

16 Übersetzung nach Otto B. Roegele, a.a.0., 313. Der lateinische Text findet sich bei Enrico Baragli, a.a.0., Tav. XXIV.

17 Vgl. Karlheinz Schmidhüs, a.a.0., $114 \mathrm{f}$. 
leuten. ${ }^{18}$ Im Rückblick auf das II. Vatikanische Konzil hat man IM nicht zu unrecht als ein im Grunde noch präkonziliäres ${ }^{19}$ Dokument charakterisiert.

Allerdings ist damit der historische Rang des Dekretes nicht ausreichend gewürdigt. Trotz der unbestreitbaren Mängel im Text bleibt die Tatsache, dass sich erstmals ein Konzil in einem eigenen Dokument mit den sozialen Kommunikationsmitteln befasst hat, von einer für die künftige kirchliche Wertschätzung der Thematik nicht hoch genug zu veranschlagenden Bedeutung.

Eine theologische Fundierung der inhaltlichen Arbeit der verschiedenen nachkonzilären Einrichtungen kirchlicher Medienarbeit konnte IM selbst freilich nicht leisten. Der wohl entscheidende Grund dafür liegt in der Tatsache, dass erst nach der sehr frühen und wohl auch überhasteten Verabschiedung des Dekretes die wichtigen ekklesiologischen Aussagen des Konzils und hier vor allem auch die Neubestimmung des Verhältnisses von Kirche und Welt in teils mühevollen Debatten auf dem Konzil erarbeitet wurden und deshalb auch noch nicht das Dekret über die sozialen Kommunikationsmittel - was von der Sache eigentlich unverzichtbar gewesen wäre - bestimmen konnten. Hier liegt der tiefere Grund für den monierten präkonziliaren Charakter des Dekretes.

Eine historisch gerechte Beurteilung wird aber berücksichtigen, dass man von IM im Nachhinein nicht etwas erwarten darf, was der Text von seiner gesamten Genese her noch nicht leisten konnte. Genau dieses Defizit hat die Pastoralinstruktion "Communio et progressio" (CeP), die schon in IM angekündigt worden war und mit deren Ausarbeitung die neu errichtete päpstliche Kommission für die Instrumente der sozialen Kommunikation unter Hinzuziehung eines internationalen Beraterstabes kurz nach Abschluss des Konzils vom Papst betraut wurde, behoben. Die Kommission erhielt den Auftrag, ein Pastoralschreiben zu entwerfen, „um alle Grundsätze und Weisungen dieses Konzils über die sozialen Kommunikationsmittel zum Erfolg zu führen“ (IM 23). ${ }^{20}$ Es hat dann

18 Nach Otto B. Roegele, a.a.0., 318. steht der Text weder auf der Höhe fachwissenschaftlicher noch theologischer Debatten. Er macht einen „antiquierten und inkompetenten Eindruck“ und besteht aus einer „unbefriedigenden Mischung pathetisch formulierter Allgemeinheiten mit gewiß gutgemeinten, aber erschreckend realitätsfernen Ermahnungen“. Vgl. Rückblick auf die Zweite Session, in: HerKorr 18 (1964), 304.

19 So z.B. Karlheinz Schmidthüs, a.a.0., 115.

20 Das motu proripo „In fructibus multis“, das die Errichtung der ständigen päpstlichen Kommission für die sozialen Kommunikationsmittel anordnete, verweist ausdrücklich auf diesen Auftrag des Konzils. Vgl. den Text des motu proprio, in: HerKorr 18 (1964), 501. 
jedoch noch Jahre gedauert, bis nach einem langwierigen Erarbeitungsprozess am 3. Juni 1971 die „Pastoralinstruktion Communio et progressio über die Instrumente der sozialen Kommunikation, veröffentlicht im Auftrage des II. Vatikanischen Ökumenischen Konzils“, wie der offizielle Titel lautet, endlich erschien. ${ }^{21}$

Wer sich über die Zielsetzungen, die das Konzil mit IM verfolgte, verständigen will, muss dafür $\mathrm{CeP}$ mit heranziehen. Das Pastoralschreiben ist als eine vom Konzil selbst gewollte Ergänzung und Weiterführung von IM zu deuten. Beide Texte gehören zusammen, und es ist nicht nur legitim, sondern notwendig, IM im Horizont von CeP zu interpretieren, wenn der Interpretation des Konzilstextes der Geist des Zweiten Vatikanischen Konzils und nicht einzig nur der Buchstabe des Dekretes zugrunde liegen soll.

\section{Die Entfaltung der kirchlichen Lehre zur sozialen Kommuni- kation: Grundlinien}

Die Verfasser von CeP haben den Auftrag aus IM weitreichend ausgelegt: Wollte IM die kirchliche „Lehre und Weisung“ (1) (doctrina et disciplina) zu den Instrumenten der sozialen Kommunikation vorlegen, die dann in dem am Ende des Dekretes angekündigten Pastoralschreiben weiter umgesetzt werden sollten, so verwirklicht $\mathrm{CeP}$ diesen Auftrag tatsächlich dadurch, dass es seinerseits noch einmal wieder - wie schon das Konzilsdekret selbst - in einer mehr allgemeinen Form „Lehrgrundsätze und pastorale Weisungen" (3) (principia doctrinae atque consilia pastoralia) formulieren will, freilich, wie die lateinische Wortwahl zeigt, jetzt mit einem deutlich pastoralen Akzent.

Konsequent haben die Verfasser von CeP diesen Auftrag unter Einbeziehung der Konzilsaussagen zum Selbstverständnis der Kirche und ihrem Verhältnis zur modernen Welt sowie im Versuch, die Eigengesetzlichkeiten der sozialen Kommunikationsmittel zu respektieren, umgesetzt. Gleichzeitig haben sie versucht, das Pastoralschreiben mit der dem Konzil voraufgegangenen Lehrtradition zur sozialen Kommunikation sowie mit nachkonziliaren lehramtlichen bzw. päpstlichen Äußerungen zu verknüpfen. Herausgekommen ist ein umfangreicher Text mit insgesamt

21 Vgl. den Bericht von Karlheinz Hoffmann zur Entstehungsgeschichte, der ausführlich zitiegrt ist bei Hans Wagner, Pastoralinstruktion „Communio et progressio" über die Instrumente der sozialen Kommunikation. Einführung und Kommentar. Trier 1971, 8-13. Anton Kochs, Zur Geschichte der Pastoralinstruktion „Communio et Progressio“, in: ComSoc 4 (1971), 293-299. 
187 Abschnitten, der zwar alle Themen aus IM aufnimmt und erweitert, sich aber in der sprachlichen und inhaltlichen Aufarbeitung dieser Themen ganz wesentlich von IM unterscheidet.

Eine kirchlich weit verbreitete Abwehrhaltung und ein schon fast konstitutionelles Misstrauen gegenüber den Medien, dass die kirchliche Tradition lange Zeit bestimmt hatte und häufig in sachfremden und moralisierenden Forderungen gegenüber den Medien zum Ausdruck kam, ließ sich mit $\mathrm{CeP}$ endgültig nicht mehr aufrechterhalten. Auch eine latent klerikale, ekklesiologische Blickverengung auf das Medienthema, die von manchen an IM noch beklagt wurde, darf mit Fug und Recht in CeP als überwunden gelten. ${ }^{22}$ Das machen bereits die ersten Sätze der Pastoralinstruktion deutlich: $\mathrm{CeP}$ beginnt nicht mehr wie noch IM mit der Feststellung des Rechtes der Kirche und der Einschärfung sittlicher Grundsätze im Umgang mit den sozialen Kommunikationsmittel, sondern stellt gleich im Eröffnungssatz fest, dass Gemeinschaft und Fortschritt der menschlichen Gesellschaft die obersten Ziele der sozialen Kommunikation sind, denen die Instrumente zu dienen haben.

In diesem Horizont werden alle Einzelthemen entfaltet. Vor allem in zwei Punkten hat CeP für die Entwicklung einer kirchenamtlichen Lehre zur sozialen Kommunikation maßgebliche Bedeutung gewonnen: Erstmals entfaltet $\mathrm{CeP}$ eine theologische Begründung sozialer Kommunikation. Das ist deshalb bedeutsam, weil damit der Begriff der sozialen Kommunikationsmittel bzw. der Instrumente der sozialen Kommunikation, den IM zwar in den lehramtlichen Sprachgebrauch eingeführt, aber nur lapidar erklärt hatte , durch $\mathrm{CeP}$ einen für den weiteren lehramtlichen Sprachgebrauch theologisch verankerten Inhalt bekam und sich damit im lehramtlichen und allgemein kirchlichen Sprachgebrauch als eigener Fachbegriff etablieren konnte. ${ }^{23}$ Auf dieser theologischen Grundlage, und das ist der zweite wichtige Punkt, reflektiert CeP neben vielen die konkrete Praxis betreffenden Fragen die unverzichtbare Bedeutung sozialer Kommunikation für die Bewahrung und Entwicklung einer freiheitlichen Gesellschaft und äußert sich auch zu den Konsequenzen dieser Position für die innerkirchliche Kommunikation. ${ }^{24}$

22 Vgl. Michael Schmolke, Zehn ideengeschichtliche Beobachtungen zur Pastoralin struktion "Communio et Progressio", in: ComSoc 4 (1971), 299-309.

23 Vgl. Franz Josef Eilers, Zum Begriff „Soziale Kommunikation“, in: ComSoc 20 (1987), 1-9.

$24 \mathrm{Zu}$ Recht kritisiert Michael Schmolke, (Zehn ideengeschichtliche Beobachtungen zur Pastoralinstruktion „Communio et progressio“, a.a.0., 307) in diesem Punkt den berühmten Kommentar zu CeP von Hans Wagner (a.a.0.). Nach Schmolke unternimmt Wagner hier den grotesken Versuch, „die kirchliche Lehrtradition zu den bürgerlichen Freiheiten und zum modernen Öffentlichkeitsbegriff umzuwerten", 


\subsection{Grundzüge einer Theologie sozialer Kommunikation}

Was ursprünglich aus christlicher Sicht unter Kommunikation zu verstehen ist, wird in CeP trinitätstheologisch verankert: „Nach christlicher Glaubensauffassung ist die Verbundenheit und die Gemeinschaft der Menschen - das oberste Ziel jeder Kommunikation - ursprünglich verwurzelt und gleichsam vorgebildet im höchsten Geheimnis der ewigen Gemeinschaft in Gott zwischen dem Vater, dem Sohn und dem Heiligen Geist, die ein einziges göttliches Leben haben" (8).

In einer schöpfungstheologischen und heilsgeschichtlichen Perspektive führt $\mathrm{CeP}$ diesen Gedanken mit Blick auf die Kommunikation unter den Menschen theologisch weiter aus. Es gehört zur Schöpfungsordnung, dass die Menschen, weil sie als Ebenbild Gottes geschaffen wurden, nach dem Urbild innergöttlicher Kommunikation ebenfalls auf Kommunikation und darin beschlossen von ihrem Wesen her auf Gemeinschaft angelegt sind. Realisiert wird diese menschliche Gemeinschaft auch durch soziale Kommunikation. „Threr ganzen Natur nach zielt die soziale Kommunikation darauf ab, dass die Menschen durch die Vielfalt ihrer Beziehungen einen tieferen Sinn für Gemeinschaft entwickeln“ (8).

Auch wenn CeP um die Gefahren der Störung und sogar Zerstörung menschlicher Gemeinschaft durch den Missbrauch der Instrumente der sozialen Kommunikation auf Grund der Sündhaftigkeit des Menschen weiß und auch darauf aufmerksam macht (vgl. 9), so überwiegt doch mit Verweis auf die Erlösung durch Christus eine optimistische, vertrauensvolle Grundhaltung gegenüber den Instrumenten der sozialen Kommunikation und damit gegenüber den Menschen, die mit ihnen als Rezipienten oder Kommunikatoren umgehen. Ihnen wird Christus als „Meister der Kommunikation“ (11), wie der lateinische Begriff „perfectus communicator" im amtlichen deutschen Text von CeP übersetzt wird, vorgestellt. Bei dieser Kennzeichnung handelt es sich freilich nicht einfach um eine erbaulich-fromme Ausschmückung des christlichen Kommunikationsverständnisses, sondern um den Versuch, das als Urbild für jede menschliche Kommunikation auf der Ebene einer immanenten Trinitätstheologie formulierte Kommunikationsmodell nun auch in der Perspektive der heilsökonomischen Trinitätstheologie christologisch so darzustellen und bibeltheologisch zu verankern, dass darin die Kommunikation Gottes mit den Menschen sowie gleichzeitig jede zwischenmenschliche Kommuni-

indem er eine Kontinuität der kirchlichen Lehrtradition behauptet, die von den kirchlichèn Verurteilungen der bürgerlichen Freiheiten, die im 18. und 19. Jahrhundert erkämpft wurden, ungebrochen bis CeP reichen soll. 
kation in dem charakterisiert wird, was ihr im Tiefsten wesentlich und $z u$ eigen ist, nämlich „Mitteilung seiner selbst in Liebe" (11).

Die Instrumente der sozialen Kommunikation werden auf diesem Hintergrund zu gottgewollten Mitteln zur Erreichung der Einheit unter den Menschen; ja, sie gehören, wie CeP in diesem Zusammenhang schon fast euphorisch formuliert „zu den wirksamsten Kräften und Möglichkeiten, die der Mensch einsetzen kann zur Stärkung der Liebe, die Ausdruck und Quelle der Gemeinschaft ist" (12). Erreicht wird dieses Ziel aber nur, wenn der Einsatz der Instrumente der sozialen Kommunikation im Dienst der Wahrheitssuche und Wahrheitsfindung sowie der Förderung des menschlichen Fortschritts geschieht (vgl. CeP 13), und zwar unter Wahrung der menschlichen Freiheit des Menschen, selbst über den $\mathrm{Ge}$ brauch der Kommunikationsmittel entscheiden zu können.

\subsection{Kommunikationsfreiheit in Gesellschaft und Kirche}

Die Instrumente der sozialen Kommunikation haben nach $\mathrm{CeP}$ die Aufgabe, das öffentliche Gespräch der Gesellschaft $z u$ ermöglichen und $z u$ befördern. Dazu aber bedarf es vor allem der Sicherung der „Öffentlichen Meinung“ und des „Rechtes auf Information“. Während IM bei diesen Themen lediglich auf "klare Gewissensgrundsätze" verwies, dabei aber völlig unzulänglich die Funktion der Kommunikationsmittel für die Gesellschaft wahrnahm, was entsprechend auch zur Verkennung der Bedeutung der Kommunikationsmittel für das Funktionieren einer freiheitliche Gesellschaft führte, entfaltet $\mathrm{CeP}$, teilweise im Rückgriff auf bereits von Pius XII. eingenommene Positionen ${ }^{25}$, ausführlich die gesellschaftliche Notwendigkeit von öffentlicher Meinung als „wesentliche(m) Ausdruck der gesellschaftlichen Natur des Menschen" (25).

Öffentliche Meinung, betont CeP, setzt Meinungsfreiheit des einzelnen voraus. „Die Freiheit der Meinungsäußerung des einzelnen ist ein Wesensbestandteil bei der Bildung von öffentlicher Meinung" (25). Die Konsequenzen aus dieser Position sind eindeutig: Verantwortung der Kommunikatoren, aber auch der Rezipienten im Bemühen um den Bestand der öffentlichen Meinung, Ablehnung jeglicher Propaganda zu Lasten des Gemeinwohls und der Beschneidung des Rechtes des Menschen auf Entscheidungsfreiheit.

Gleiches gilt für das Recht der Menschen auf Information. Während IM sich nur sehr allgemein zu diesem Thema äußert und im gleichen Atemzug mit der Feststellung des Rechts auf Information auf die damit

25 Vgl. Giselbert Deussen, Die Pastoralinstruktion auf dem Hintergrund der päpstlichen Lehrtradition, in: ComSoc 4 (1971), 310-322. 
verbundenen ethischen Grundsätze, nämlich Verpflichtung zur Wahrheit und - in den Grenzen, die die menschliche Rücksichtnahme erfordert auch Vollständigkeit aufmerksam macht (vgl. IM 5), wählt $\mathrm{CeP}$ einen anderen Weg: In ausdrücklicher Rückbindung an die kirchliche Tradition und an das Konzil legt CeP den eindeutigen Schwerpunkt auf die Explikation des Rechtes auf Information als Recht sowohl des einzelnen Bürgers als auch als Forderung vom Gemeinwohl her.

Ganz deutlich versucht CeP hier mit dem Medienthema grundlegend Anschluss an die Errungenschaften der modernen pluralistischen Gesellschaften und ihren Werthaltungen zu bekommen. Das ist in CeP wirklich neu gegenüber IM. Interessant ist in diesem Zusammenhang eine Formulierung, nach der gerade die sogenannten pluralistischen Gesellschaften sehr wohl um den Wert der Kommunikationsfreiheit ihrer Bürger wissen, und diese mit besonderen Gesetzen schützen. Wörtlich heißt es dann noch: „Die Allgemeine Erklärung der Menschenrechte hat die Kommunikationsfreiheit als Grundforderung herausgestellt. Diese schließt notwendig die Freiheit der Instrumente der sozialen Kommunikation ein “ (46).

Dass ein solches Bekenntnis zur Kommunikationsfreiheit auch für die innerkirchliche Kommunikation nicht folgenlos bleiben kann, wird von $\mathrm{CeP}$ explizit angesprochen. IM hatte sich hier weitgehend ausgeschwiegen. CeP geht einen anderen Weg. Auch die Kirche bedarf nach $\mathrm{CeP}$ sowohl in ihren Beziehungen nach außen als auch für das innerkirchliche Leben der sozialen Kommunikation. Mit einem Zitat aus einer Rede von Pius XII wird festgestellt: „Dem Leben der Kirche würde etwas fehlen, wenn es in ihr an öffentlicher Meinung mangelte. Die Schuld daran fiele auf Hirten und Gläubige“ (115).

Auch Katholiken besitzen in der Kirche „wirklich die Freiheit der Meinungsäußerung“" (116). CeP hält für den Binnenraum der Kirche an dem theologisch begründeten Prinzip der Kommunikationsfreiheit grundsätzlich fest und fordert die Bischöfe auf, dafür zu sorgen, „dass sich innerhalb der Kirche auf der Basis der Meinungs- und Redefreiheit der Austausch legitimer Ansichten lebendig entfaltet" (116). Ausdrücklich ermutigt CeP zur Freiheit des Gespräches innerhalb der Kirche. Die Einheit werde dadurch nicht bedroht, sondern im Gegenteil sogar befördert. Voraussetzung dabei ist, dass „bei aller Meinungsverschiedenheit die Liebe bestimmend bleibt, und jeder von dem Wollen beherrscht ist, das Gemeinsame zu wahren und die Zusammenarbeit zu sichern" (116).

Wie allgemein gesellschaftlich, so gilt auch innerkirchlich, dass sich öffentliche Meinung nur dort entfalten kann, wo auch das Recht auf Information beachtet wird. Deshalb fordert $\mathrm{CeP}$ von der Kirche, auf die 
Praxis der Geheimhaltung, die gerade in der Kirche eine lange Tradition hat, weitestgehend zu verzichten. „Wenn kirchliche Stellen Nachrichten zurückhalten oder nicht in der Lage sind $z \mathfrak{u}$ informiëren, öffnen sie schädlichen Gerüchten Tür und Tor, anstatt die Wahrheit ans Licht zu fördern“ (121). Geheimhaltung ist einzig dort zulässig, wo bei Veröffentlichung Ruf und Ansehen eines Menschen oder Rechte von einzelnen bzw. Gruppen verletzt würden.

In dieser Deutlichkeit hatte bisher noch kein kirchliches Dokument zur Kommunikationsfreiheit innerhalb der Kirche Stellung bezogen und hat es seitdem auch nicht wieder getan.

Das Presseecho auf die Veröffentlichung war in einer ersten Phase zurückhaltend bis kritisch. ${ }^{26}$ Es hat noch eine Weile gedauert, bis CeP geradezu zum Grundlagendokument katholischer Medienarbeit avancierte.

\section{Die Entfaltung der kirchlichen Lehre zur sozialen Kommuni- kation: Konkretisierungen}

\subsection{Soziale Kommunikation im neuen Kirchenrecht}

Im alten CIC gab es keine Normen zur sozialen Kommunikation sondern lediglich einige Canones (1384-1405) zur vorbeugenden Bücherzensur und zum unterdrückenden Bücherverbot. Um möglichst alle schädlichen und bösen Einflüsse, die von Druckerzeugnisse ausgehen könnten, von den Katholiken fernzuhalten, versuchte man ein von einem Gesetzeszaun gesichertes, katholisches Ghetto zu errichten. ${ }^{27}$ Die rechtlichen Normen spiegeln dabei durchgängig eine Abwehrhaltung. Ein typischer Ausdruck dieser Mentalität war der sog. Index der verbotenen Bücher, der erst 1966 abgeschafft worden ist und bis dahin Jahrhunderte lang das geistige Klima in der Kirche im Umgang mit Büchern bestimmt hatte. Wenn die in IM und CeP grundgelegten Weisungen im Umgang mit den Instrumenten der sozialen Kommunikation rechtlich normiert werden sollten, konnte man sich nicht einfach auf gesetzestechnische Anpassungen der Canones 1384-1405 beschränken. Es bedurfte vielmehr einer umfassenden Neuordnung. Diese Neuordnung wurde im neuen CIC (1983) vorgenommen.

26 Vgl. Franz Josef Eilers, „Communio et Progressio“ im Spiegel der Presse, in: ComSoc 5 (1972), 108-117.

27 Vgl. Heinz Mussinghoff, Neues Kirchenrecht und Kommunikation, in: ComSoc 18 (1985), 143-157. 
Neben einer Reihe von Einzelnormen in verschiedenen Teilen des CIC (1983) gibt es im Buch III „Verkündigungsdienst der Kirche“ einen eigenen Abschnitt über soziale Kommunikationsmittel, insbesondere Bücher (de instrumentis communicationis socialis et in specie de libris), der die Canones 822-832 umfasst.

Neu gegenüber dem alten CIC ist die ausdrückliche Feststellung des Rechts auf freie Meinungsäußerung aller Christgläubigen in der Kirche in Can $212 \& 3 \mathrm{im}$ neuen CIC. ${ }^{28}$ Es heißt dort: „Entsprechend ihrem Wissen, ihrer Zuständigkeit und ihrer hervorragenden Stellung haben sie das Recht und bisweilen sogar die Pflicht, ihre Meinung in dem, was das Wohl der Kirche angeht, den geistlichen Hirten mitzuteilen und sie unter Wahrung der Unversehrtheit des Glaubens und der Sitten und der Ehrfurcht gegenüber den Hirten und unter Beachtung des allgemeinen Nutzens und der Würde der Personen den übrigen Gläubigen kundzutun."

Eine unbegrenzte Meinungsfreiheit, das zeigt der Gesetzestext deutlich, gibt es in der Kirche nicht. Alle Meinungsäußerungen müssen die Unversehrtheit von Glaube und Sitte wahren, sie müssen von einer achtungsvollen Haltung gegenüber den Hirten getragen sein, und sie müssen das kirchliche allgemeine Wohl sowie die Würde der Person beachten. Das Recht auf freie Meinungsäußerung ist grundlegend durch die für alle Christgläubigen verbindliche Pflicht zu Glaubensgehorsam und zu Gesetzesgehorsam begrenzt. ${ }^{29}$ Trotz dieser Begrenzungen bleibt aber festzuhalten, dass der CIC (1983) das einseitig hierarchisch-eindimensionale Kommunikationsmodell von oben nach unten mit der Proklamation der Anerkennung der freien Meinungsäußerung innerhalb der Kirche aufgekündigt hat. Dass sich der konkrete Gebrauch der sozialen Kommunikationsmittel im Übrigen an den allgemein geltenden Pflichten und Rechten der Christgläubigen zu orientieren hat, ergibt sich aus der Sache selbst.

Eine unmittelbare Anknüpfung an IM 3 findet sich im neuen CIC ebenfalls in der Feststellung des Rechtes der Kirche, alle Arten von Kommunikationsmittel anzuwenden und also auch zu besitzen (Can 822 , $\S 1)$. Dieses Recht gründet im Missions- und Verkündigungsauftrag der Kirche. Es verpflichtet alle in der Kirche, zu einem von menschlichem und christlichem Geist belebten Umgang und Einsatz der Medien in der Pastoral $(\S 2,3)$. Das Recht und die Pflicht der Hirten, auf die Unversehrtheit der Glaubens- und die Sittenlehre auch auf dem Gebiet der sozialen Kommunikationsmittel zu achten, wird ausdrücklich in Can 823 hervorgehoben.

$28 \mathrm{Vgl}$. Heinrich Reinhardt, MKCIC Can. 212.

29 Vgl. Heinz Mussinghoff, a.a.0., 146. 


\subsection{Ausbildung der Priester in sozialer Kommunikation}

Ein wichtiger, allerdings in der Praxis der Priesterausbildung bedauerlicherweise, noch längst nicht ausreichend wirksam gewordener Impuls zur Rezeption von IM und CeP ist von der Kongregation für das katholische Bildungswesen ausgegangen. Im März 1986 hat sie „Leitlinien für die Ausbildung der künftigen Priester in den Medien der sozialen Kommunikation" 30 herausgegeben. Das besondere Ziel dieser Leitlinien besteht in einer "zugleich menschlich-kulturellen und christlich-moralischen Ausbildung der künftigen Priester im rechten, zunächst persönlichen und dann pastoralen Gebrauch sämtlicher Medien der sozialen Kommunikation“. 31

In mehrfacher Hinsicht sind die Leitlinien bemerkenswert. Ganz auf der Linie von CeP räumen sie den Massenmedien eine hohe Bedeutung für die Kommunikation der Menschen untereinander ein und formulieren auf diesem Hintergrund dann differenziert die unterschiedlichen Erfordernisse für eine zeitgemäße Priesterausbildung. Misstrauen oder eine Abwehrhaltung gegenüber der Massenkommunikation ist nirgends in den Leitlinien spürbar. Wohl aber wird auf die Komplexität des Bereichs der sozialen Kommunikation verwiesen, die sachgerecht nur in einer interdisziplinären Forschungsperspektive verstanden werden kann. Gerade die Anerkenntnis dieses Zusammenhangs in den Leitlinien verhindert eine sachfremde Moralisierung oder vorschnelle Instrumentalisierung im Umgang mit den Massenmedien. Einer solchen Mentalität setzen die Leitlinien die Forderung nach medienpädagogischer Kompetenz der Priester entgegen. Die Priester sollen auf der Grundlage solider Fachkenntnisse die besonderen Chancen für Apostolat und Verkündigung nutzen, die die modernen Medien bieten können. Dass in diesem Zusammenhang von den künftigen Priestern auch ein disziplinierter persönlicher Gebrauch der Medien erwartet wird und im Seminar dazu auch erzogen werden muss, kann nicht als Bewahrpädagogik gedeutet werden. Vielmehr wird darin aus geistlichen Gründen von den künftigen Priestern eine bestimmte Askese im Medienkonsum gefordert, die für alle Mediennutzer, und nicht nur für künftige Priester, heute vielleicht sogar aktueller ist als vor gut $25 \mathrm{Jahren}$, als die Leitlinien formuliert wurden.

So gesehen gehören die Leitlinien sicher $z u$ jenen nachkonziliaren Texten, in denen für einen zentralen Lebensbereich der Kirche die Lehraussagen zur Sozialen Kommunikation gewissenhaft umgesetzt wurden.

30 Rom 1986.

31 Leitlinien, Anhang II, Vorbemerkung, a.a.0. 
Die Umsetzung dieser Leitlinien in die Praxis dürfte auch gegenwärtig noch als Herausforderung an die Priesterausbildung begriffen werden.

\subsection{Neue Herausforderungen}

Wohl aufgeschreckt durch eine explosionsartige Zunahme von gewalthaltigen und pornographischen Darstellungen in den Medien veröffentlichte der Päpstliche Rat für die sozialen Kommunikationsmittel am 7. Mai 1989 ein Dokument mit dem Titel „Pornographie und Gewalt in den Kommunikationsmedien. Eine pastorale Antwort“". ${ }^{32}$ Deutlich spürbar wird gerade in dem Teil des Dokuments, der sich mit den unterstellten Wirkungen von Pornographie und Gewalt in den Medien befasst, das Erschrecken über eine Medienwirklichkeit, die in deutlichen Widerspruch zu der grundlegend wohlwollenden Wahrnehmung der Medien in CeP steht.

Die Ursache für das Anwachsen von Pornographie und Gewalt in den Medien sieht der Rat in einer allgemein wachsenden „moralischen Freizügigkeit“ der Menschen, „die im Suchen nach persönlicher Befriedigung um jeden Preis ihre Wurzel hat. Verbunden damit ist eine Art von verzweifelter Leere, die Sinnenrausch als einziges Glück, das Menschen erreichen können, betrachtet" (19). Der Rat diagnostiziert eine auf Sexualität fokussiert konsumistische Lebensform als den entscheidenden Nährboden für das Anwachsen von Pornographie und Gewalt in den Medien, die von einem ungezügelten Profitdenken im Bereich der Kommunikationsindustrie gesteuert und ausgebeutet wird, in manchen Ländern sogar im Bündnis mit dem organisierten Verbrechen (vgl. 20).

Welche Antwort gibt der Rat, um das diagnostizierte Problem zu lösen? Grundsätzlich steht fest, dass das Recht auf freien Ausdruck und freien Austausch von Informationen nicht angetastet werden darf. Ebenso aber müssen das Recht auf Privatsphäre, auf Wahrung des öffentlichen Anstands und der Schutz der Grundwerte geachtet werden. Auf diesem Hintergrund werden die unterschiedlichen Verantwortungsträger angesprochen: So wird den berufsmäßigen Kommunikatoren beispielsweise dringend empfohlen, ,gemeinsam ethische Normen für die Kommunikationsmedien $z \mathfrak{u}$ formulieren und anzuwenden" (23). Eltern werden auf ihre Erziehungsaufgaben hingewiesen. Mit Berufung auf $\mathrm{CeP}$ werden sie daran erinnert, dass man bei der Bildung des Menschen "mehr durch begründete Erklärung als durch Verbot erreicht" (24, CeP 64). Die Jugendlichen werden zu eigenverantwortlichem moralischen Handeln auf-

32 Hrsg. vom Sekretariat der Deutschen Bischofskonferenz, Bonn 1989 (Arbeitshilfen 71) 
gefordert, das breite Publikum soll sich wirksam öffentlich äußern, und die staatlichen Autoritäten werden auf ihre Aufgaben des Jugendschutzes und ihre Verpflichtung, für das Gemeinwohl zu sorgen, hingewiesen. In einer „Zeit des Permissivismus“ (29) sieht die Kirche für dich selbst die Aufgabe, klare ethische Grundsätze zu formulieren, die Zusammenarbeit auf breiter Ebene zu suchen und sich für eine kirchliche Medienerziehung einzusetzen.

Die pastorale Antwort besteht also in einer Erinnerung an grundlegende sittliche Verpflichtungen und in der Betonung medienpädagogischer Aufgaben. Damit steht auch dieser Text des Päpstlichen Rates grundsätzlich auf der Linie, die vom Konzil gegenüber den Medien eingeschlagen wurde, auch wenn gegenüber $\mathrm{CeP}$ eine Ernüchterung in der Beurteilung der Medien unverkennbar ist. Diese Ernüchterung führt aber nicht in eine von Misstrauen diktierte Abwehrhaltung zurück. Auch werden die Medien nicht in die Rolle des Sündenbocks für einen beklagten Verfall moralischer Werte gedrängt. Der Weg der Kirche, den IM und besonders $\mathrm{CeP}$ eingeschlagen haben, bleibt gültig. „Eine bloß Zensur ausübende Haltung der Kirche den Medien gegenüber ist endlich weder ausreichend noch angebracht. Die Kirche sollte sich statt dessen um ein ständiges Gespräch mit verantwortlichen Kommunikatoren bemühen, um sie bei ihrer Arbeit zu ermutigen und ihnen Hilfe anzubieten, wo dies notwendig ist oder erbeten wird" (30).

Unter dieser grundlegenden Zielsetzung stehen auch die übrigen in den letzten 15 Jahren veröffentlichten lehramtlichen Texte zur sozialen Kommunikation. ${ }^{33}$

1992, also gut zwanzig Jahre nach CeP hat der Päpstliche Rat für die Sozialen Kommunikationsmittel eine neue Pastoralinstruktion unter dem Titel „Aetatis Novae“ (AN) veröffentlicht. ${ }^{34}$ Die technische Entwicklung der Medien seit CeP kam einem revolutionären Umbruch gleich und verlief weiter in einem rasanten Tempo. Mit dem Zusammenbruch des politischen Systems des osteuropäischen und sowjetischen Kommunismus hatte sich die Welt zudem auch politisch radikal verändert. Die Medien

33 Mit Blick auf die innerkirchliche Kommunikationsfreiheit sind in dieser Zeit lehramtliche Versuche, diese Freiheit restriktiv auszulegen, allerdings unübersehbar. Dazu: Helmuth Rolfes, Lehramt, Theologie und Öffentlichkeit. Überlegungen zum Öffentlichkeitsverständnis der „Instruktion über die kirchliche Berufung des Theologen", in: ComSoc 23 (1990), 207-216. Ders., Soziale Kommunikation und Wahrheitsverwaltung. Überlegungen zu Aetatis Novae Nr. 10 über die Medien im Dienste der kirchlichen Gemeinschaft, in: ComSoc 25 (1992), 269-275.

34 Hrsg. vom Sekretariat der deutschen Bischofskonferenz, Bonn 1992 (Arbeitshilfen 98).Vgl. das Themenheft 3/4: 20 Jahre „communio et progressio" von ComSoc 24 (1991). 
hatten dabei eine machtvolle Rolle gespielt. AN will auf dem Hintergrund dieser Entwicklungen IM und besonders auch $\mathrm{CeP}$ weiterdenken und gleichzeitig gegenüber den neuen Herausforderungen eine Ermutigung aussprechen. Dabei zitiert AN die theologische Sicht der Sozialen Kommunikation aus CeP und hebt entsprechend die Dienstfunktion der Medien für das gesellschaftliche Leben hervor. Deutlich akzentuiert wird in diesem Zusammenhang dann aber von AN vor allem die besondere $\mathrm{Be}$ deutung der Medien für die Kommunikation der Kirche mit der Welt und für die Neu-Evangelisierung. Erkennbar zurückhaltender als in $\mathrm{CeP}$ wird die innerkirchliche Kommunikationsfreiheit in einem eigenen Abschnitt (10) angesprochen. Im Vordergrund stehen jetzt kirchenrechtliche und disziplinäre Gesichtspunkte. ${ }^{35}$

AN zeichnet sich dadurch aus, dass neben der Anerkenntnis der positiven Wirkungen auch die unabsehbar negativen Folgen in der Entwicklung der Medienlandschaft angesprochen werden. Die Pastoralinstruktion fordert auf diesem Hintergrund dazu auf, die ethischen und moralischen Kriterien $\mathrm{zu}$ verdeutlichen, die in den menschlichen und christlichen Werten angelegt sind und die auch in den einzelnen Feldern der sozialen Kommunikation Gültigkeit haben. Bereits IM und $\mathrm{CeP}$ hatten bis in Institutionalisierungen hineinreichende Forderungen zur Medienpastoral in einem umfassenden Sinne aufgestellt. AN nimmt diese Forderungen auf und empfiehlt den Diözesen und den Bischofskonferenzen dringend, „dass in alle ihre Pastoralpläne der Problembereich der sozialen Kommunikation eingebracht wird“ (21). Die Dringlichkeit dieser Forderung wird durch den Hinweis auf eine Kritik des Papstes an einer oftmals noch unter den Verantwortlichen der Kirche vorherrschenden Auffassung unterstrichen, wonach die Massenmedien in der Verkündigung eine eher untergeordnete Rolle spielen. „Diese Situation erfordert eine Richtigstellung“ (20). AN lässt es aber nicht bei allgemeinen Forderungen und Appellen bewenden. In einem eigenen, mehrseitigen Anhang stellt AN sehr differenziert „Elemente eines Pastoralplanes für soziale Kommunikation" vor, die als Leitlinien für die Erarbeitung von Medienpastoralplänen der Ortskirchen dienen sollen. Eine gewissenhafte Umsetzung dieser Leitlinien in pastorales Planen und Handeln dürften viele Bistümer - auch in Deutschland - eher wohl noch als Aufgabe vor sich haben. Sie würde auf jeden Fall zu einer Steigerung der Kompetenz im Gebrauch und Umgang der Medien unter den in der Kirche Verantwortlichen beitragen.

35 Vgl. Helmuth Rolfes, Soziale Kommunikation und Wahrheitsverwaltung, a.a.0. 


\subsection{Ethik und Soziale Kommunikation}

Immer vordringlicher wurde angesichts der Medieneñtwicklung der jüngsten Vergangenheit die Formulierung ethischer Grundsätze für die soziale Kommunikation. Das lässt sich unter anderem an der Zunahme der Publikationen zu Fragen der Medienethik in der nichtkirchlichen Publizistik ablesen. Auch das kirchliche Lehramt hat auf diese Entwicklung reagiert. So erschienen in relativ kurzen zeitlichen Abständen gleich drei Verlautbarungen des Päpstlichen Rates, die in ihrem Titel das Stichwort „Ethik" tragen. ${ }^{36}$

Die Medien erfordern keine "neue Ethik“, wohl aber die Anwendung grundlegender ethischer Prinzipien auf die neue Situation, wie es im Dokument „Ethik in der sozialen Kommunikation" heißt (28). Die Frage, wie ethische Prinzipien und Normen im Medienbereich Anwendung finden können, ist freilich nicht einfach zu beantworten. Der Anwendungsbereich betrifft ja nicht nur die Inhalte der Kommunikation und die Kommunikationsprozesse, sondern auch den Bereich der Struktur und des Systems der Medien, der oft mit weitreichenden, brisanten politischen und ökonomischen Fragen verknüpft ist. Einfache Lösungen sind hier nicht $\mathrm{zu}$ haben. Um zu ethisch relevanten Erkenntnissen zu kommen, bedarf es deshalb eines breiten Diskurses mit grundsätzlich allen, die am Mediengeschehen beteiligt sind.

Für diesen Diskurs will die Kirche mit ihren Ethikdokumenten Überlegungen vorlegen, die das Nachdenken und den Dialog fördern sollen (vgl. 20). Zunächst geht es in den Dokumenten zur Medienethik darum, einige ethische Grundprinzipen in Erinnerung zu rufen, die als Fundament aller medienethischen Überlegungen angesehen werden. In diesem Sinne lautet ein erstes Grundprinzip: „Der Mensch und die Gemeinschaft der Menschen sind Ziel und Maßstab für den Umgang mit den Medien“ (21). Ergänzt wird dieses Prinzip durch ein zweites Grundprinzip: „Das Wohl der Menschen lässt sich nicht unabhängig vom Gemeinwohl der Gemeinschaft verwirklichen, der sie angehören“ (22). Diese beiden Grundprinzipien werden in den Ethik-Verlautbarungen des Päpstlichen Rates ausdrücklich in einer globalen Perspektive ausgelegt. Konsequenterweise zählt deshalb auch die Einforderung einer weltweiten Kommunikationsgerechtigkeit zu den zentralen Anliegen der kirchlichen Medienethik. „Es gibt einen dringenden Bedarf an Gerechtigkeit auf internationaler Ebene“ (22). Den kirchlichen Ethikdokumenten zu Fragen der so-

36 Hrsg. vom Sekretariat der Deutschen Bischofskonferenz, Ethik in der Werbung, Bonn 1917 (Arbeitshilfen 135); Ethik in der Sozialen Kommunikation, Bonn 2000 (Arbeitshilfen 153); Ethik im Internet, Bonn 2002 (Arbeitshilfen 163). 
zialen Kommunikation kommt das besondere Verdienst $\mathrm{zu}$, im gesellschaftlichen Diskurs über Medienethik eine Sichtweise zu akzentuieren, die sich an der Forderung einer weltweiten Solidarität orientiert und aus dieser Orientierung dann konkrete Handlungsperspektiven entwirft.

Ausdrücklich thematisiert und konkretisiert wird die Frage der Kommunikationsgerechtigkeit im Dokument „Ethik im Internet“. ${ }^{37}$ Wie kein anderes Medium eröffnet das Internet einen globalen Informationsaustausch und prinzipiell für jeden weltweite Kommunikationsmöglichkeiten. Der Zugang zum Internet ist allerdings zwischen den reichen Industrieländern und den armen Entwicklungsländern sowie auch innerhalb einzelner Gruppen in einzelnen Ländern höchst ungerecht verteilt. Es besteht eine „digitale Kluft“ (10). Diese digitale Kluft, nach der auf der einen Seite die Nutznießer der neuen Medien stehen und auf der anderen Seite all jene, die keinen oder nur einen ungenügenden Zugang haben, muss überwunden werden, damit die Globalisierungsprozesse der gesamten Menschheit zu Gute kommen und nicht einzig einigen wohlhabende Eliten (vgl. 10).

Die Faszination über die neuen Kommunikationsmöglichkeiten, die das Internet bietet und die auch für den Verkündigungsauftrag der Kirche genutzt werden müssen ${ }^{38}$, darf nicht darüber hinwegtäuschen, dass sich viele ohnehin schon vorhandene ethische Probleme in der sozialen Kommunikation durch das Internet in verschärfter Weise stellen: die Gefahr eines (westlichen) Kulturimperialismus zu Lasten eines gerade im Zuge der Globalisierung unverzichtbaren interkulturellen Dialogs; die Gefährdung der Meinungsfreiheit und des freien Ideenaustausches durch totalitäre Regime oder einflussreiche Einzelgruppen; die aufgrund der spezifischen Natur des Internets veränderte Herausforderung bezüglich Recherche, Vollständigkeit und Genauigkeit für die journalistische Arbeit im Internet. Hier besteht nach Auffassung des Päpstlichen Rates aktueller Handlungsbedarf.

Auch wenn über die ethische Missbilligung bestimmter Internetseiten (z.B. Kinderpornographie, Aufruf zum Hass usw.) und sog. Internetstraftaten (z.B. Verbreitung von Computerviren oder Einbruch in Datenbanken und Diebstahl von Daten) kein Zweifel besteht, so zeigen doch diese Sachverhalte, dass für das Internet die überkommenen rechtlichen Steuerungsmechanismen, z. B. zum Schutz der Privatsphäre, von Sicherheit und Vertraulichkeit von Daten, von Urheberrechten oder geistigen

37 Veröffentlicht als Begleitdokument zu „Ethik im Internet“, a.a.O.

$38 \mathrm{Vgl}$. das zusammen mit dem Dokument „Ethik im Internet" veröffentlichte Begleitdokument „Kirche und Internet", in dem diese neuen Möglichkeiten aufgelistet und empfohlen werden. 
Eigentums, völlig unzureichend funktionieren. Gleiches gilt für die Durchsetzung einer auch nur minimalen ethischen Norm. Nach Auffassung des Päpstlichen Rates wird deshalb für die Zukunft eine von ethischen Grundüberzeugungen getragene supranationale und grenzüberschreitende Zusammenarbeit unverzichtbar sein, um einen „internationalen Konsens" zu erarbeiten, der es ermöglicht, die angesprochenen Probleme besser in den Griff zu bekommen (vgl. 17).

Die entscheidenden Anknüpfungspunkte für die ethischen Überlegungen in den angesprochenen Dokumenten lassen sich bereits in $\mathrm{CeP}$ nachlesen. Alle ethischen Grundsätze stützen sich nach $\mathrm{CeP}$ nämlich auf „den Vollbegriff von der Würde des Menschen“ (14). ${ }^{39}$ In diesem Punkt ging $\mathrm{CeP}$ übrigens über IM hinaus. IM war weitgehend bei der Darstellung und Einschärfung von Gewissengrundsätzen im Umgang mit den Medien stehen geblieben; $\mathrm{CeP}$ bemüht sich dagegen um eine Rückbindung solcher Grundsätze in schöpfungstheologische und heilsgeschichtliche Überlegungen unter ausdrücklicher Anerkenntnis der Eigengesetzlichkeit der sozialen Kommunikation. Genau diesen Weg gehen auch die Ethik-Verlautbarungen des Päpstlichen Rates, wenn sie versuchen - oft auf der Negativfolie von Missständen - positive ethische Grundsätze zu formulieren. Die besonderen Beiträge, die die Kirche in die medienethischen Diskussionen einbringt, bestehen „in einer Auffassung von der menschlichen Person und ihrer unvergleichlichen Würde, ihren unverletzbaren Rechten und in einer Auffassung von der menschlichen Gemeinschaft, deren Glieder durch die Tugend der Solidarität beim Streben nach dem gemeinsamen Wohl aller untereinander verbunden sind" (Ethik in der sozialen Kommunikation 30). Das ist der grundlegende Bezugspunkt für die Erörterung einzelner Probleme in den Ethik-Verlautbarungen des Päpstlichen Rates. Er markiert außerdem die maßgebliche Lehrkontinuität mit $\mathrm{CeP}$.

\section{Vierzig Jahre nach Inter Mirifica: Gibt es eine kirchenamtliche Lehre der sozialen Kommunikation?}

Das Verhältnis der Kirche zu den Medien hat sich mit dem Zweiten Vatikanischen Konzil grundlegend verändert. Die Kirche hat, mit IM beginnend, dann vor allem in $\mathrm{CeP}$ die Grundlinien einer kirchenamtlichen Lehre zur sozialen Kommunikation vorgelegt, die in den folgenden lehr-

39 Vgl. Helmuth Rolfes, Christlicher Glaube und Medienethik. Der Beitrag von Theologie und Kirche zum medienethischen Gespräch der Gesellschaft, in: Matthias Karmasin (Hrsg.), Medien und Ethik, Stuttgart 2002, 238-259. 
amtlichen Dokumenten wirksam geworden ist. Erstmals wurde der Versuch unternommen, eine ausdrücklich theologische Theorie der sozialen Kommunikation zu formulieren. Seit dieser Zeit hat sich der Begriff der sozialen Kommunikation in der amtskirchlichen und theologischen Sprache als eigener Fachbegriff etabliert. Ganz auf der Linie und im Geiste des Konzils hat diese theologische Klärung dazu geführt, die unverzichtbare Bedeutung sozialer Kommunikation für den Erhalt und die Weiterentwicklung einer freiheitlichen Gesellschaft auch theologisch zu begreifen und $z \mathfrak{u}$ entfalten. Damit wurden zentrale Orientierungslinien für eine nachkonziliare Lehre der sozialen Kommunikation aufgezeigt, die sich durch alle nachfolgenden lehramtlichen Dokumente zur sozialen Kommunikation mit unterschiedlicher Akzentuierung hindurchziehen. Unter dieser Rücksicht ist die Frage, ob es eine kirchenamtliche Lehre der sozialen Kommunikation gibt, eindeutig zu bejahen.

Nun hat sich Medienlandschaft seit dem Zweiten Vatikanischen Konzil geradezu revolutionär verändert, und sie wird sich auch weiterhin verändern. Neue Entwicklungen und Herausforderungen erfordern deshalb auch von der Kirche neue Antworten, die sich nicht einfach aus einmal eingenommenen Positionen ableiten lassen. So gesehen müssen alle kirchlichen Texte zur sozialen Kommunikation sowohl in theologischer als auch medienspezifischer Hinsicht gemäß der vom Konzil geforderten Option für eine freiheitliche und gerechte Gesellschaft weitergeschrieben werden. Das dies auch tatsächlich geschieht, zeigen die nachkonziliaren Dokumente. Alle Dokumente der jüngeren Vergangenheit zeigen darüber hinaus, dass die durch $\mathrm{CeP}$ begründete positive und konstruktive Sichtweise auf die Medien auch dort nicht verworfen wird, wo ganz konkret schwere Missstände und Fehlentwicklungen angeprangert werden.

Für die gegenwärtigen Debatten lassen sich zwei Schwerpunkte ausmachen. Gegenüber der lehrmäßigen Betonung der allgemeinen Bedeutung der sozialen Kommunikationsmittel für eine freiheitliche Gesellschaft rückt aus kirchenamtlicher Sicht seit geraumer Zeit die Forderung immer mehr in den Vordergrund, die modernen Medien für die Evangelisierung sowie bei den unterschiedlichen Verkündigungsaufgaben der Kirche einzusetzen und entsprechende pastorale Planungsstrategien $z \mathbf{u}$ entwerfen. Fundament solcher Bemühungen muss freilich das bleiben, was lehrmäßig seit dem Konzil Gültigkeit hat. Dass die kirchliche Realität sich solchen Forderungen gegenüber oft noch als resistent erweist, zeigen die bisher kaum umgesetzten Leitlinien zur Ausbildung der Priester in sozialer Kommunikation und der Pastoralplan aus AN. Auch bei der Nutzuffg des Internets dürfte die Entwicklung trotz bereits vielfältig vorhandener Aktivitäten noch in den Anfängen stecken.

Ein zweiter Schwerpunkt liegt in der kirchlichen Forderung nach einer 
entfalteten Medienethik. Diese Forderung lässt sich schon in IM erkennen, hat aber erst durch $\mathrm{CeP}$ eine theologische Richtung bekommen und steht zur Zeit auf der Agenda kirchenamtlicher Verlautbarungen zur sozialen Kommunikation. Sie ist als Reaktion auf eine Entwicklung der Medien- und Kommunikationslandschaft zu verstehen, in der die Kommunikationsfreiheit auf vielfältige Weise gefährdet und oft auch missachtet wird. Im Diskurs der Gesellschaft zu medienethischen Fragen kommt der Kirche zukünftig vermutlich vermehrt die Aufgabe zu, die ethischen Implikationen der kirchlichen Lehre der sozialen Kommunikation zu Gehör zu bringen. In der Abarbeitung an konkreten Problemen der Mediengesellschaft wäre dann die Lehre der sozialen Kommunikation selbst weiter $\mathrm{zu}$ entwickeln. 